\title{
High-resolution computed tomography and pulmonary function in children with common variable immunodeficiency
}

\author{
L. van Zeggeren*, A.A.J.M. van de Ven\#, S.W.J. Terheggen-Lagro", O.M. Mets*, \\ F.J. Beek*, J.M. van Montfrans ${ }^{\#}$ and P.A. de Jong*
}

ABSTRACT: High-resolution computed tomography (HRCT) may be useful to monitor lung disease in children with common variable immunodeficiency disorder (CVID). We evaluated interobserver agreement and correlation with pulmonary function tests (PFTs) for automated quantification and visual scoring of air trapping and airway wall thickening on HRCT in paediatric CVID patients.

In a cohort of 51 children with CVID, HRCT was analysed visually and automated for presence of air trapping and airway wall thickening. PFTs were expressed as \% predicted. Disease duration, physician-diagnosed pneumonias and antibiotic prophylaxis were recorded.

Interobserver agreement for automated airway wall thickening was good with an intra-class correlation coefficient of $\mathbf{0 . 8 8}$, compared with $\mathbf{0 . 5 1}$ for visual scoring. Presence of air trapping on HRCT correlated significantly with PFTs and disease duration, but was not associated with previous pneumonias. Airway wall thickening did not correlate significantly with PFTs or disease duration and was not associated with previous pneumonias or prophylactic antibiotic use.

In children with CVID disorders, HRCT air trapping measurements are significantly correlated with PFTs and disease duration. Quantitative air trapping is a feasible and promising technique for small airway disease quantification that may be applied to monitor (silent) disease progression in CVID.

KEYWORDS: Immunodeficiency syndrome, paediatric respiratory diseases, radiology

ommon variable immunodeficiency (CVID) is a heterogeneous immunodeficiency syndrome characterised by B-cell dysfunction, resulting in hypogammaglobulinaemia. Because of the low levels of immunoglobulins (Ig), CVID patients are prone to developing recurrent lower respiratory tract infections [1, 2]. It has been reported that $90 \%$ of CVID patients suffer from one or more episodes of lower respiratory tract infections throughout their life [3], and structural pulmonary high-resolution computed tomography (HRCT) abnormalities are reported in up to $93 \%$ of patients $[1,2]$. For the prevention of irreversible airway damage, CVID patients are treated with Ig replacement therapy and antibiotics as a prophylaxis or when needed for respiratory infections. Despite adequate treatment, progression of pulmonary disease occurs in a considerable number of patients and, recently, an overall mortality rate of $25 \%$ was reported 15 yrs after diagnosis $[4,5]$. Because pulmonary complications are commonly seen and therapeutic options are available, it is important to monitor the presence and progression of lung disease in CVID patients [2]; this can be done clinically with laboratory tests, cultures, pulmonary function tests (PFTs) and imaging techniques. HRCT can demonstrate disease in asymptomatic CVID patients. This is important, as disease can progress without any notable episode of pneumonia, described as "silent progression" $[6,7]$. Recently, a CVID-related lung disease HRCT score was reported, which aimed to quantify the structural disease component [2]. However, visual scores are time-consuming and associated with interobserver variation. The interobserver variation was substantial, especially for visual airway wall thickening scores (intra-class correlation coefficient 0.51 ). Validated software is available to quantify airway wall dimensions [8, 9] and software initially developed for emphysema quantification [10-12] could be used to quantify end-expiratory air trapping.

The aims of the current study were to evaluate the relationship of visual scores and quantitative measures of HRCT-diagnosed air trapping and airway wall thickening with PFTs in a cohort of children with CVID(-like) disease, and to determine whether observer agreement for airway wall
AFFILIATIONS

*Depts of Radiology,

\#Paediatric Immunology and

Infectious Diseases, and

"Paediatric Pulmonology, University

Medical Center Utrecht and

Wilhelmina Children's Hospital,

Utrecht, The Netherlands.

CORRESPONDENCE

P.A. de Jong

Dept of Radiology

University Medical Center Utrecht

HP E.01.132

Heidelberglaan 100

3584 CX Utrecht

The Netherlands

E-mail: pimdejong@gmail.com

Received:

Nov 072010

Accepted after revision:

May 252011

First published online:

June 092011

European Respiratory Journal Print ISSN 0903-1936 Online ISSN 1399-3003 
measurements can be improved by using automated quantitative techniques.

\section{MATERIAL AND METHODS \\ Study population}

Children with clinically important humoral immunodeficiencies underwent structured follow-up at our outpatient paediatric clinic (University Medical Center Utrecht and Wilhelmina Children's Hospital, Utrecht, the Netherlands). Follow-up included pulmonary evaluation with HRCT and PFTs. We evaluated 54 children with CVID(-like) disease who were treated with Ig replacement therapy and showed significant clinical improvement after initiation of therapy. Three patients were excluded from the study because the raw HRCT data were not stored properly in one and software failed in two, thus the study population consisted of 51 children. These 51 HRCT scans were performed between June 2008 and June 2009 and were all obtained in clinically stable patients, otherwise the scan was postponed. $33(65 \%)$ children were diagnosed with CVID according to the European Society for Immunodeficiencies (ESID)/Pan-American Group for Immunodeficiency (PAGID) criteria [13], while 18 (35\%) did not meet all of the ESID criteria for CVID. These 18 patients were defined as CVID-like (symptomatic selective antibody deficiency in combination with IgA deficiency and/or IgG subclass deficiency), but showed clinical and immunological phenotypes indistinguishable from CVID patients. For antibiotic prophylaxis, co-trimoxazole was used in each protocol. This retrospective investigation was approved by the ethical review board and informed consent was waived.

\section{HRCT scanning protocol}

Chest HRCT scanning was performed, conforming to a dedicated low-dose volumetric protocol, on a 16-detector-row computed tomography (CT) scanner (Brilliance-16; Philips, Cleveland, $\mathrm{OH}$, USA). Scans were obtained during both inspiration and expiration using a breath-hold instruction. Inspiratory scans were acquired in a caudocranial direction with a collimation of $16 \times 0.75 \mathrm{~mm}$, pitch 0.9 , rotation time $0.5 \mathrm{~s}, 90 \mathrm{kVp}$ and milli-amperage per second depending on body weight (range 16-60 mA $\cdot \mathrm{s}^{-1}$ ). Expiratory scans were acquired in a caudocranial direction with a collimation of $16 \times 0.75 \mathrm{~mm}$, pitch 1.2 , rotation time $0.4 \mathrm{~s}, 90 \mathrm{kVp}$ and $11 \mathrm{~mA} \cdot \mathrm{s}^{-1}$. The expiratory scan was obtained at end expiration.

\section{HRCT measurements}

In two patients the expiration HRCT scan was obtained at insufficient exhalation (visual judgement); therefore, air trapping scores and measurements were reported for 49 HRCT scans. The visual scores for airway wall thickening and air trapping have been described previously [2]. Two blinded independent observers scored the extent of air trapping on a scale from 0 (absent) to 5 ( $>80 \%$ of the volume of a lobe affected), the extent of airway wall thickening on a scale from 0 (absent) to 3 ( $>67 \%$ of the volume of a lobe affected), and the average and maximum severity of severe airway wall thickening per lobe. Airway wall thickening severity was defined as mild (airway wall thickness to accompanying pulmonary artery ratio $0.33 / 0.5$ ), moderate (airway wall thickness to artery ratio $0.5 / 1$ ) and severe (airway wall thickness to artery ratio $>1$ ). Abnormalities were scored for each of the six lobes including the lingula. The range for air trapping was 0 (absent in all lobes) to 30 ( $>80 \%$ involvement of all lobes). For airway wall thickening for each lobe, the most severe score (maximum 3 ) was added to the average score (maximum 3 ), and the sum was multiplied by the extent score (maximum 3 ). This led to a range of 0 (no airway wall thickening) to 108 (all airways severely thickened). The HRCT scores were expressed on a scale of $0-100 \%$. The presence of ground-glass opacities was recorded.

Validated custom software was used for the automated measurements (EmphylxJ; University of British Columbia, Vancouver, BC, Canada) [8, 9, 11, 12]. Airway wall area (WA) and lumen area (LA) dimensions were quantified for the right upper lobe apical bronchus (RB1) and the area of the right lung at this level was measured (fig. 1). The airway quantification of RB1 was performed by two independent observers in order to calculate interobserver agreement. Wall area was expressed as a percentage of total airway area: $100 \times \mathrm{WA} /(\mathrm{WA}+\mathrm{LA})=\mathrm{WA} \%$. As $\mathrm{WA} \%$ is not constant with age, it was corrected for age by using the formula: WA $\%$ age $=$ WA $\%+(18-$ age $\times 0.72)$. This formula was obtained from airway measurements published previously [14]. WA was also normalised to body size by dividing WA by body surface area (using the formula of MOSTELLER [15], WABSA) and by right lung area (WAlung area).

For airway wall quantification, all perpendicular airways at every tenth CT slice were measured. We obtained perimeter of the inner airway lumen $(\mathrm{Pi})$ and WA. $\sqrt{ }$ WA was plotted on the vertical axis against $\mathrm{Pi}$, resulting in a linear relationship. The linear regression formula was obtained for each patient and, by using this formula, the WA at a Pi of $10 \mathrm{~mm}$ was calculated (WAPi10).

For air trapping quantification, the lung parenchyma was segmented at every tenth expiratory HRCT slice and the total number of voxels and voxels $<-850$ Hounsfield units $(\mathrm{HU})$ were quantified (fig. 2). This cut-off value has been described previously [16]. The number of voxels below the cut-off was expressed as a percentage of the total number of voxels: ATHU-850 $=100 \times$ number of voxels $<-850 \mathrm{HU} /$ total number of voxels. The HU at the 15th percentile was also calculated (AT15).
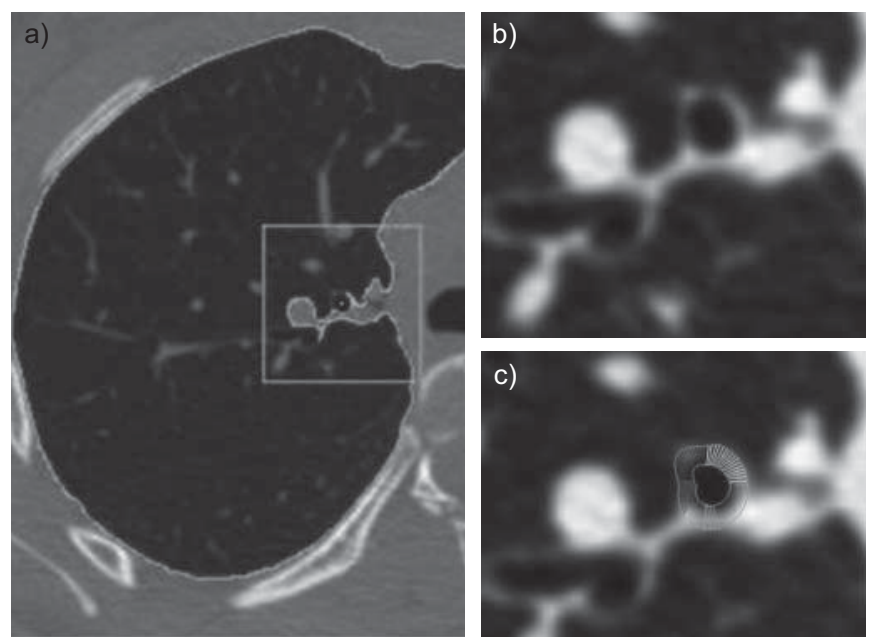

FIGURE 1. Example of automated airway measurement technique of right upper lobe apical bronchus (RB1) on an inspiratory high-resolution computed tomography slice. a) Right lung area at RB1 is identified. b, c) Enlarged area of the area highlighted by the box in a. The airway wall is then automatically defined and can be adjusted manually if required (c). 

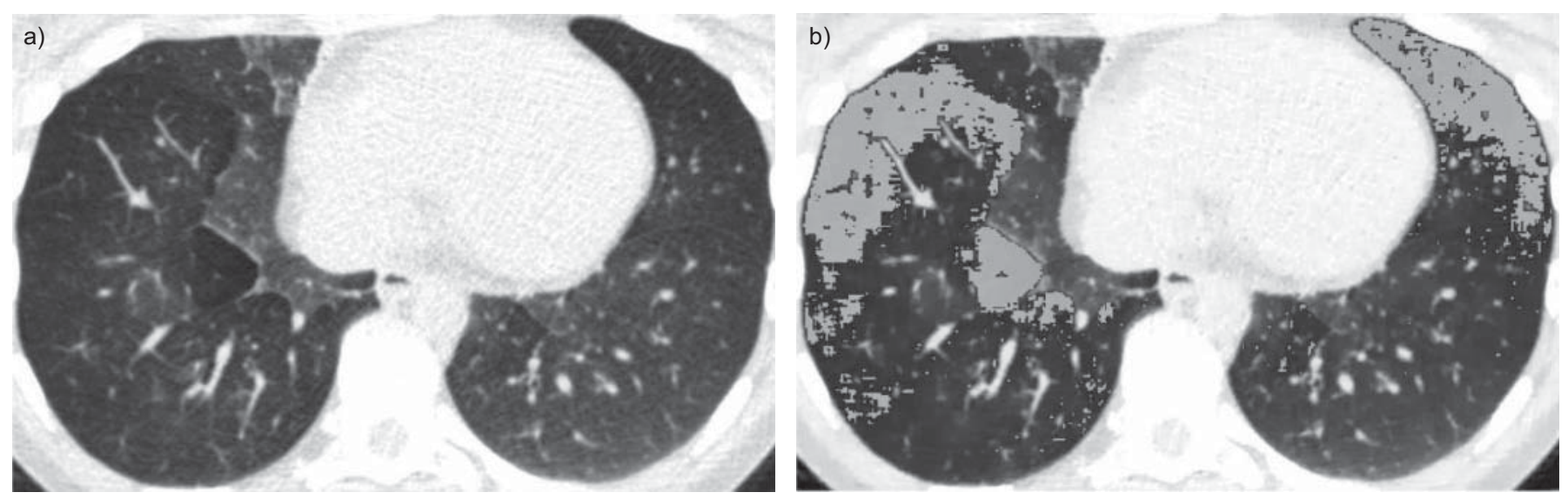

FIGURE 2. Demonstration of the air trapping quantification technique on an expiratory high-resolution computed tomography (HRCT) slice. a) The original expiratory HRCT slice demonstrates differences in density reflecting air trapping. b) The voxels with a density below a certain cut-off value, in this example $<-850$ Hounsfield units, are highlighted (grey shaded areas) and quantified.

\section{Pulmonary function tests}

Two $(4 \%)$ children were unable to perform spirometry and four $(8 \%)$ could not perform plethysmography. Spirometric measurements and body plethysmographic measurements were obtained according to the American Thoracic Society criteria [17, 18]. Spirometric measurements included forced vital capacity (FVC), forced expiratory volume in $1 \mathrm{~s}$ (FEV1) and maximum midexpiratory flow at $25-75 \%$ of FVC (MMEF). Body plethysmography included residual volume (RV) and total lung capacity (TLC). Measurements were expressed as percentage of predicted values. Reference formulas from a Dutch cohort of healthy children were used [19]. Two ratios were calculated and expressed as a percentage: RV/TLC and FEV1/FVC.

\section{Statistical analysis}

Interobserver agreement for RB1 WA\%, airway wall thickening score and air trapping score [2] was evaluated using scatter plots with a line of identity, and intra-class correlation coefficients were calculated. This correlation took account the distance to the line of identity. An intra-class correlation $>0.8$ represented good agreement. The relationship between PFTs, disease duration and HRCT parameters was visualised using scatter plots and Spearman rank correlations were calculated. Unpaired sample ttests were used to determine whether HRCT measurements differed between patients who experienced physician-diagnosed pneumonia and those who had not, or those who used antibiotic prophylaxis. Analyses were performed using SPSS 15.0 (SPSS Inc., Chicago, IL, USA). Statistical significance was set at $\mathrm{p}<0.05$. Data are reported as mean $\pm \mathrm{SD}$ and (range), unless otherwise stated.

\section{RESULTS}

\section{Patient characteristics and pulmonary function}

The mean \pm SD (range) age of the 51 children (38 males and 13 females) at the time of HRCT was $13.1 \pm 3.5$ (6-18) yrs. Mean disease duration was $6.5 \pm 3.5(0.1-13)$ yrs. 27 (53\%) out of the 51 children were diagnosed with one or more pneumonia before the HRCT was performed. A total of $22(43 \%)$ children were using regular antibiotic prophylaxis prior to the HRCT. On average, children with CVID had a preserved lung function with an FEV1 of $96.3 \pm 12 \%$ (66.6-129.1\%). Further details are presented in table 1.

\section{HRCT scores and measurements}

The interobserver agreement for the automated RB1 WA\% measurement was good with an intra-class correlation coefficient of 0.88 . The interobserver agreement for the visual scores has been previously reported and was 0.51 for airway wall thickening and 0.78 for air trapping [2]. WA\%age was $72.5 \pm 7.4 \%$ (54.9-92.4\%). WAPi10 was $4.3 \pm 0.6(3.5-6.0) \mathrm{mm}$. Ground-glass opacities were present in seven (14\%) out of the 49 children (table 1).

\section{Correlation between HRCT, PFTs, disease duration and antibiotic use}

Visual air trapping score correlated significantly with FVC, FEV1, MMEF and FEV1/FVC\%, but not with RV/TLC\% and disease duration (tables 2 and 3). ATHU-850 correlated significantly with MMEF and, when patients with ground-glass attenuation on HRCT $(n=7,14 \%)$ were excluded, this correlation became stronger and also significant for AT15 (table 2). Disease duration was also significantly associated with quantitative air trapping measures (table 3; fig. 3).

For airway wall thickening, neither visual scoring nor automated quantitative measures were associated with decreased pulmonary function (table 2) or longer disease duration (table 3). Of note, the significant correlations of FEV1, MMEF and RV/TLC $\%$ with quantitative airway measurements were in the opposite direction, i.e. a worse lung function was associated with a lower wall thickness. HRCT measurements did not differ between patients who experienced physician-diagnosed pneumonia and those who had not, or those who used antibiotic prophylaxis (table 4).

\section{DISCUSSION}

Airway disease is an important complication of CVID and HRCT and PFTs are gaining important complementary roles in monitoring it. We showed that in children with CVID disorders, HRCT air trapping measurements are significantly correlated with PFTs and disease duration, and that interobserver agreement for HRCT airway wall thickening assessment can be improved by using automated quantitative techniques compared to visual scores. Thus, automated quantitative HRCT, especially for air trapping, may prove useful for monitoring disease progression in CVID patients. 


\begin{tabular}{|c|c|}
\hline Patient characteristic & \\
\hline Patients n & 51 \\
\hline Age yrs & $13.1 \pm 3.5$ \\
\hline Male sex & $38(75)$ \\
\hline CVID & $33(65)$ \\
\hline CVID-like disease & $18(35)$ \\
\hline Disease duration yrs & $6.5 \pm 3.5$ \\
\hline $\begin{array}{l}\text { One or more physician diagnosed } \\
\text { pneumonia before HRCT }\end{array}$ & $27(53)$ \\
\hline Antibiotics prophylaxis & $22(43)$ \\
\hline Body surface area $\mathrm{m}^{2}$ & $1.4 \pm 0.3$ \\
\hline FEV 1 \% pred & $96.3 \pm 12$ \\
\hline FVC \% pred & $94.6 \pm 11$ \\
\hline MMEF \% pred & $100.8 \pm 28.9$ \\
\hline $\mathrm{FEV}_{1 / \text { FVC } \%}$ & $90 \pm 6.8$ \\
\hline RV/TLC \% & $22.7 \pm 6.8$ \\
\hline \multicolumn{2}{|l|}{ Visual HRCT scores } \\
\hline Air trapping present & $43(84)$ \\
\hline Airway wall thickening present & $31(61)$ \\
\hline Ground-glass attenuation present & $7(14)$ \\
\hline \multicolumn{2}{|l|}{ Quantitative HRCT measurements } \\
\hline Wall area $\mathrm{mm}^{2}$ & $24 \pm 12$ \\
\hline Wall area \% & $69 \pm 7.2$ \\
\hline WABSA $\mathrm{mm}^{2} \cdot \mathrm{m}^{-2}$ & $18 \pm 12$ \\
\hline WAlung area $\mathrm{mm}^{2} \cdot$ voxels $\mathrm{s}^{-1}$ & $0.85 \times 10^{-5} \pm 0.413 \times 10^{-5}$ \\
\hline WA\%age \% & $72.5 \pm 7.4$ \\
\hline Number of measured airways at $10 \mathrm{~mm}$ & $87 \pm 28.5$ \\
\hline WAPi10 $\mathrm{mm}^{2}$ & $43 \pm 6$ \\
\hline ATHU-850 \% & $9.3 \pm 7.7$ \\
\hline AT15 HU & $-803 \pm 53$ \\
\hline ATHU-850 corrected for ground glass \% & $9.2 \pm 7.7$ \\
\hline AT15 corrected for ground glass HU & $-803 \pm 53$ \\
\hline
\end{tabular}

Data are presented as mean $\pm \mathrm{SD}$ or $\mathrm{n}(\%)$, unless otherwise stated. CVID: common variable immunodeficiency; HTCT: high-resolution computed tomography; FEV1: forced expiratory volume in $1 \mathrm{~s}$; \% pred: \% predicted; FVC: forced vital capacity; MMEF: maximum mid-expiratory flow at $25-75 \%$ of FVC RV: residual volume; TLC: total lung capacity; WABSA: wall area normalised to body size; WAlung area: wall area normalised to right lung area at right upper lobe apical bronchus level; WA\%age: wall area percentage corrected for age; WAPi10: wall area at a perimeter of the inner airway lumen of $10 \mathrm{~mm}$; HU: Hounsfield units; ATHU-850: number of voxels $<-850 \mathrm{HU}$ as percentage of total number of voxels; AT15: $\mathrm{HU}$ at 15th percentile.

For air trapping, we demonstrated that visual, as well as quantitative, HRCT measurements correlate significantly with PFTs and disease duration. For visual scoring of air trapping, previous studies have also found a significant correlation with PFTs in CVID patients [20]. These data and our data indicate that HRCT-diagnosed air trapping is related to relevant airway obstruction in these patients. The advantage of our study is the use of quantitative techniques of air trapping that have not previously been employed in CVID. Using this technique, we demonstrated that the presence of air trapping correlates the strongest with MMEF, a functional measure of small airways disease. A previous study by THICKETT et al. [21] suggests that airway disease in CVID, as in other diseases such as cystic fibrosis, may start in the small airways. It is likely, that although we have no pathological verification, quantitative air trapping is a sign of small airway disease that occurs in childhood and increases with longer duration of the disease. The correlations with PFTs are stronger for visual air trapping scores than for quantitative measures. Several reasons could account for this. First, the presence of ground-glass opacity apparently had an effect on the quantitative measurements. It may be that more sophisticated air trapping measures that combine inspiratory and expiratory $\mathrm{CT}$, such as the relative volume change, can be used to overcome this problem [22]. Secondly, differences in depth of expiration introduces noise; it is possible that human observers detect an air trapping pattern at a variety of expiration levels while computerised analysis is more dependent on a stable expiration between patients. For that reason, spirometer gating or more sophisticated quantification techniques might be advantageous [23, 24]. However, quantitative air trapping measurements correlated more strongly with disease duration than the visual scores. Based on these results, we can neither advocate visual nor quantitative HRCT air trapping measurements for disease assessment in CVID, although the quantitative measurements have the advantage of being fully automated.

For airway wall thickening, we demonstrated that neither visual nor quantitative airway wall measurements correlated with pulmonary function or disease duration, although this does not necessarily exclude the possibility of monitoring disease progression. Quantitative airway wall measurements had not previously been employed in CVID. We tried several strategies to "normalise" the airway dimensions by age, body size and lung size for RB1 and we calculated WA at a lumen perimeter of $10 \mathrm{~mm}$ for all perpendicular airways as a normalisation. Nevertheless, we were unable to demonstrate a significant correlation with lung function or disease duration and, therefore, cannot recommend a specific automated measurement or calculation of airway wall thickening. We also could not explain this lack of correlation by use of prophylactic co-trimoxazole in some patients. Other studies have reported a significant correlation between airway wall thickening and pulmonary function in CVID [25], and it is generally accepted that recurrent pulmonary infections and inflammation typically cause bronchial wall thickening. An explanation for the discrepancy with our results might be that we performed HRCT in clinically stable patients, while in most other CVID studies, HRCT scans were performed for a clinical indication. It may also be that in children with CVID, mainly peripheral airway walls are involved. With routine CT technology, these airways cannot be directly visualised and for visible airways the quantification is most accurate in airways with a diameter $>2 \mathrm{~mm}$ as airway wall dimensions are overestimated in smaller visible airways with most software. It may well be that the airway walls of the larger airways that we could quantify in our study are spared in stable CVID children. We have no clear explanation for the significant correlations of FEV1, MMEF and RV/TLC\% with quantitative airway wall measurements in the unexpected direction, and we were unable to identify any CVID-specific pathological studies of airway abnormalities that could clarify this result. In conclusion, although large airway wall thickening appears to be an acute disease marker in CVID, it appears that it is not a good disease marker in stable children with CVID, where airway wall disease is probably limited to the small airways; although, it cannot be excluded that longitudinal studies of 


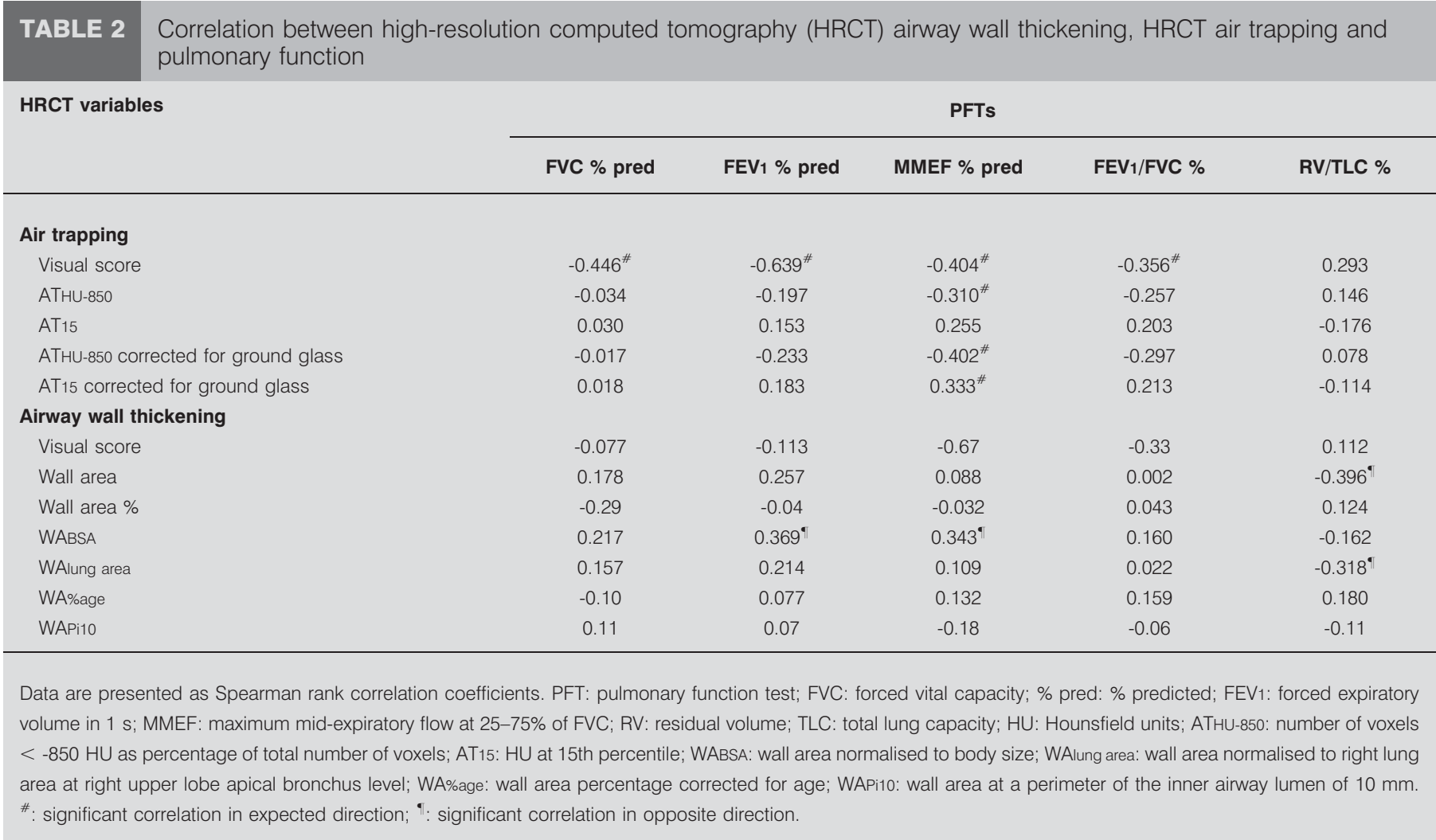

airway wall thickening will be able to detect disease progression in large airway walls of stable CVID children.

We evaluated the reproducibility of HRCT measurements of air trapping and airway wall thickening. It has previously been described [2] that air trapping and airway wall thickening are common findings on visually judged HRCT scans of CVID patients, although, especially for airway wall thickening, interobserver variation was considerable. Herein, we showed that quantitative, automated measurements of airway wall thickness

\begin{tabular}{llc} 
TABLE 3 & $\begin{array}{l}\text { Correlation between disease duration, high- } \\
\text { resolution computed tomography (HRCT) airway } \\
\text { wall thickening and HRCT air trapping }\end{array}$ \\
\cline { 2 - 3 } \begin{tabular}{l} 
HRCT variables \\
\cline { 2 - 3 }
\end{tabular} & \multicolumn{2}{c}{ Disease duration } \\
\hline Spearman rank & p-value \\
Visual score air trapping & -0.061 & 0.676 \\
Visual score airway wall thickening & 0.089 & 0.536 \\
Wall area percentage & -0.061 & 0.671 \\
WABSA & -0.038 & 0.797 \\
WA\%age & -0.274 & 0.052 \\
ATHU-850 & 0.417 & 0.004 \\
AT15 & -0.382 & 0.008 \\
\hline
\end{tabular}

WABSA: wall area normalised to body size; WA\%age: wall area percentage corrected for age; HU: Hounsfield units; ATHU-850: number of voxels < -850 HU as percentage of total number of voxels; AT15: $\mathrm{HU}$ at 15th percentile. substantially improve interobserver agreement. We also demonstrated that fully automated quantification of air trapping (without interobserver variation) is possible and can be achieved by using a simple cut-off at $-850 \mathrm{HU}$ or by assessing the HU value at the 15th percentile on the expiratory HRCT. This gain in interobserver agreement by using quantitative techniques is promising for the long-term monitoring of airway abnormalities in patients with CVID. It has been shown that quantitative airway wall measurements in other diseases, such as cystic fibrosis, can detect disease progression [26], and it is plausible that longitudinal studies could prove HRCT to be able to detect (silent) disease progression in CVID patients as well, and eventually may improve clinical management in these patients.

In the present study, we did not quantify bronchiectasis, which would be another interesting disease measure in these children, as bronchiectasis already occur in childhood [2]. For quantification of bronchiectasis the lumen area must, however, be normalised by using the area of the accompanying pulmonary artery (ratio of airway lumen area to pulmonary artery area). To our knowledge, no commercially available software exists for pulmonary artery area measurements. Another possibility for bronchiectasis quantification is the measurement of airway lumen tapering, but for such measurements, three-dimensional software is required.

Our study has some limitations. First, given our cross-sectional study design, we cannot determine whether HRCT can detect (silent) disease progression in patients with stable PFTs and absence of symptoms. As longitudinal studies are required to assess this relationship, we plan to re-evaluate our cohort after 2 yrs. Secondly, although inadequate expiration may affect 

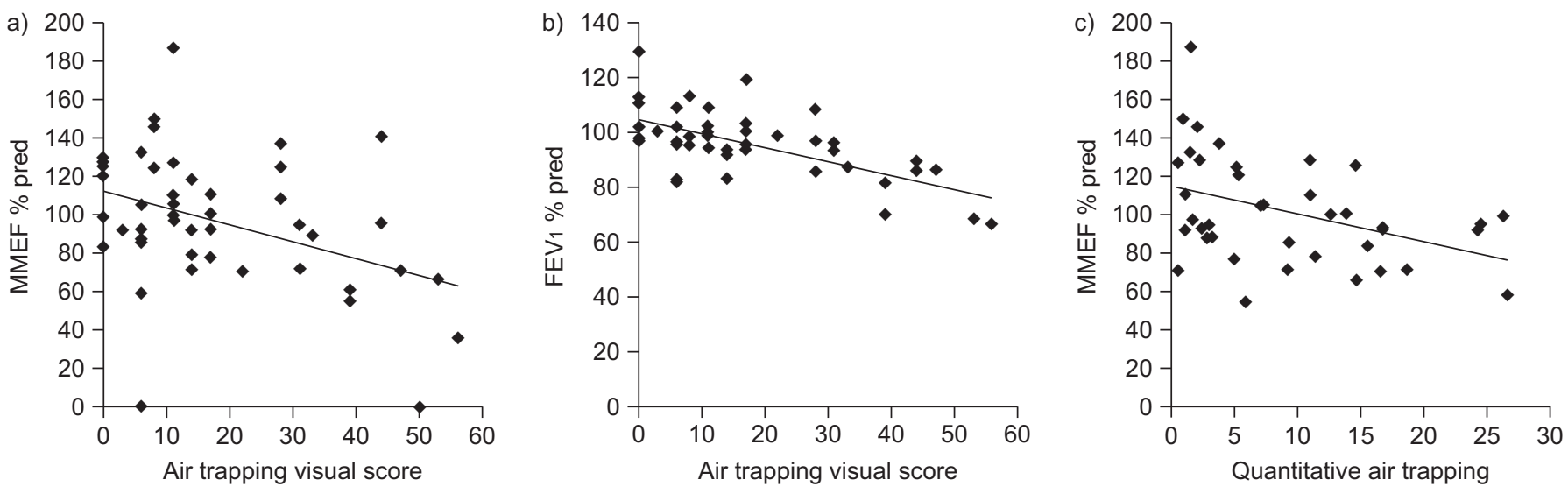

FIGURE 3. Scatter plots illustrating significant correlations between visual scores for air trapping and a) maximum mid-expiratory flow at 25-75\% of forced vital capacity (MMEF) and b) forced expiratory volume in $1 \mathrm{~s}$ (FEV1). c) Quantitative measurements of air trapping for cut-off value of $-850 \mathrm{HU}$ in patients with no ground-glass attenuation on high-resolution computed tomography and MMEF. Correlation coefficients are presented in table 2. \% pred: \% predicted.

HRCT measurements, we did not control the level of inspiration and expiration by spirometry-gated HRCT, for example. Nevertheless, since our aim was for a clinically applicable test and spirometer-gated scanning is not widely available, its absence strengthens the generalisability of our results. Thirdly, no pathological correlate was available for our HRCT measurements and, to our knowledge, CVID-specific pathology studies of airway abnormalities have not been published to date. Fourthly, we could not find a significant difference in HRCT measurements between children who had and those who had not experienced a physician-diagnosed pneumonia. This may be due to the retrospective evaluation of these pneumonias from the patient files, leading to pneumonias that were not recorded in our study for several children. Finally, our study lacks control HRCT scans of "healthy" children and, therefore, we are unable to determine a specific cut-off level for pathology. However, at this stage, our choice for quantitative or visual HRCT analysis for future research would be based on interobserver agreement and correlation with lung function.

In conclusion, we report that automated quantitative HRCT measurements improve interobserver agreement for airway wall measurements compared with visual scores in a cohort of paediatric CVID patients. Whether wall measurements in larger airways will prove useful in stable CVID children is questionable as these measurements are not significantly associated with PFTs,

TABLE 4 Physician-diagnosed pneumonias and high-resolution computed tomography (HRCT) Scores

HRCT variables

\begin{tabular}{ccc} 
Physician diagnosed pneumonia before HRCT \\
\hline Yes & No & $\begin{array}{c}\text { p-value } \\
\text { difference }\end{array}$
\end{tabular}

Patients $\mathbf{n}$

Visual score air trapping

Visual score airway wall thickening

ATHU-850

AT15

ATHU-850 corrected for ground glass

AT15 corrected for ground glass

Wall area $\mathrm{mm}^{2}$

Wall area \%

WABSA

WAlung area $\times 10^{-5}$

WA\%age

WAPi10

$\begin{array}{ccc}27 & 24 & \\ 21 \pm 19 & 13 \pm 11 & 0.108 \\ 4 \pm 6 & 3 \pm 4 & 0.462 \\ 12 \pm 12 & 9 \pm 8 & 0.280 \\ -814 \pm 57 & -798 \pm 56 & 0.341 \\ 12 \pm 12 & 10 \pm 9 & 0.604 \\ -808 \pm 60 & -806 \pm 51 & 0.932 \\ 0.21 \pm 0.09 & 0.27 \pm 0.15 & 0.127 \\ 68 \pm 8 & 70 \pm 7 & 0.296 \\ 0.15 \pm 0.05 & 0.20 \pm 0.15 & 0.183 \\ 0.78 \pm 0.37 & 0.93 \pm 0.45 & 0.225 \\ 71 \pm 8 & 74 \pm 7 & 0.297 \\ 0.42 \pm 0.06 & 0.44 \pm 0.06 & 0.468\end{array}$

\begin{tabular}{ccc}
\multicolumn{3}{c}{ Antibiotic prophylaxis $^{\#}$} \\
\hline Yes & No & $\begin{array}{c}\text { p-value } \\
\text { difference }\end{array}$
\end{tabular}

Data are presented as mean \pm SD, unless otherwise stated. Difference tested with unpaired sample t-tests. HU: Hounsfield unit; ATHU-850: number of voxels < -850 HU as percentage of total number of voxels; AT15: HU 15th percentile; WABSA: wall area normalised to body size; WAlung area: wall area normalised to right lung area at right upper lobe apical bronchus level; WA\%age: wall area percentage corrected for age; WAPi10: wall area at a perimeter of the inner airway lumen of 10 mm. ${ }^{\#}$ : co-trimoxazole per protocol. 
disease duration or number of pneumonias. Conversely, our study cannot exclude that quantitative airway wall measurements can detect disease progression or acute disease changes. Our quantitative air trapping data suggest that, in stable children with CVID, mainly the walls of small airways are involved and quantitative air trapping is a feasible and promising technique for small airways disease quantification, which may be applied to monitor (silent) disease progression in CVID as early detection of complications is of crucial importance to prevent long-term morbidity, especially in paediatric patients.

\section{STATEMENT OF INTEREST}

A statement of interest for A.A.J.M. van de Ven can be found at www. erj.ersjournals.com/site/misc/statements.xhtml

\section{REFERENCES}

1 Touw CM, van de Ven AA, de Jong PA, et al. Detection of pulmonary complications in common variable immunodeficiency. Pediatr Allergy Immunol 2010; 21: 793-805.

2 van de Ven AA, van Montfrans JM, Terheggen-Lagro SW, et al. A CT scan score for the assessment of lung disease in children with common variable immunodeficiency disorders. Chest 2010; 138: 371-379.

3 Martínez García MA, de Rojas MD, Nauffal Manzur MD, et al. Respiratory disorders in common variable immunodeficiency. Respir Med 2001; 95: 191-195.

4 Chapel H, Lucas M, Lee M, et al. Common variable immunodeficiency disorders: division into distinct clinical phenotypes. Blood 2008; 112: 277-286.

5 Healy MJ. Hypogammaglobulinaemia in the United Kingdom. XII. Statistical analyses: prevalence, mortality and effects of treatment. Spec Rep Ser Med Res Counc (GB) 1971; 310: 115-123.

6 Kainulainen L, Varpula M, Liippo K, et al. Pulmonary abnormalities in patients with primary hypogammaglobulinemia. J Allergy Clin Immunol 1999; 104: 1031-1036.

7 Plebani A, Soresina A, Rondelli R, et al. Clinical, immunological, and molecular analysis in a large cohort of patients with X-linked agammaglobulinemia: an Italian multicenter study. Clin Immunol 2002; 104: 221-230.

8 Nakano Y, Muro S, Sakai H, et al. Computed tomographic measurements of airway dimensions and emphysema in smokers. Correlation with lung function. Am J Respir Crit Care Med 2000; 162: 1102-1108.

9 Nakano Y, Whittall KP, Kalloger SE, et al. Development and validation of human airway analysis algorithm using multidetector row CT. Proceedings of SPIE 2002; 4683: 460-469.

10 Müller NL, Staples CA, Miller RR, et al. "Density mask". An objective method to quantitate emphysema using computed tomography. Chest 1988; 94: 782-787.
11 Coxson HO, Mayo JR, Behzad H, et al. Measurement of lung expansion with computed tomography and comparison with quantitative histology. J Appl Physiol 1995; 79: 1525-1530.

12 Coxson HO, Rogers RM, Whittall KP, et al. A quantification of the lung surface area in emphysema using computed tomography. Am J Respir Crit Care Med 1999; 159: 851-856.

13 Conley ME, Notarangelo LD, Etzioni A. Diagnostic criteria for primary immunodeficiencies. Representing PAGID (Pan-American Group for Immunodeficiency) and ESID (European Society for Immunodeficiencies). Clin Immunol 1999; 93: 190-197.

14 de Jong PA, Long FR, Wong JC, et al. Computed tomographic estimation of lung dimensions throughout the growth period. Eur Respir J 2006; 27: 261-267.

15 Mosteller RD. Simplified calculation of body surface area. N Engl J Med 1987; 317: 1098.

16 Busacker A, Newell JD Jr, Keefe T, et al. A multivariate analysis of risk factors for the air-trapping asthmatic phenotype as measured by quantitative CT analysis. Chest 2009; 135: 48-56.

17 Standardization of Spirometry, 1994 Update. American Thoracic Society. Am J Respir Crit Care Med 1995; 152: 1107-1136.

18 Stocks J, Godfrey S, Beardsmore C, et al. Plethysmographic measurements of lung volume and airway resistance. Eur Respir J 2001; 17: 302-312.

19 Koopman M, Zanen P, Kruitwagen CL, et al. Reference values for paediatric pulmonary function testing: the Utrecht dataset. Respir Med 2011; 105: 15-23.

20 Gregersen S, Aaløkken TM, Mynarek G, et al. High resolution computed tomography and pulmonary function in common variable immunodeficiency. Respir Med 2009; 103: 873-880.

21 Thickett KM, Kumararatne DS, Banerjee AK, et al. Common variable immune deficiency: respiratory manifestations, pulmonary function and high-resolution CT scan findings. QJM 2002; 95: 655-662.

22 Matsuoka S, Kurihara Y, Yagihashi K, et al. Quantitative assessment of air trapping in chronic obstructive pulmonary disease using inspiratory and expiratory volumetric MDCT. AJR Am J Roentgenol 2008; 190: 762-769.

23 Robinson TE, Leung AN, Moss RB, et al. Standardized highresolution $\mathrm{CT}$ of the lung using a spirometer-triggered electron beam CT scanner. AJR Am J Roentgenol 1999; 172: 1636-1638.

24 Goris ML, Zhu HJ, Blankenberg F, et al. An automated approach to quantitative air trapping measurements in mild cystic fibrosis. Chest 2003; 123: 1655-1663.

25 Gharagozlou M, Ebrahimi FA, Farhoudi A, et al. Pulmonary complications in primary hypogammaglobulinemia: a survey by high resolution CT scan. Monaldi Arch Chest Dis 2006; 65: 69-74.

26 de Jong PA, Nakano Y, Hop WC, et al. Changes in airway dimensions on computed tomography scans of children with cystic fibrosis. Am J Respir Crit Care Med 2005; 172: 218-224. 